\title{
RADIOLOGICAL CALCIFICATION OF INTERVERTEBRAL DISCS IN CHILDREN
}

BY

\author{
I. S. WALLMAN \\ From the Princess Margaret Hospital for Children, Perth, Western Australia
}

(RECEIVED FOR PUBLICATION JULY 27, 1956)

It seems strange that a child should develop calcification of the intervertebral discs, a process which is generally regarded as degenerative in nature, but such is the case in the rare disorder first described by Báron in 1924 . There are only 16 cases of this condition in children described in the literature, the most complete description being that of Silverman (1954) who reported seven cases and considered that it was more common than the number of reported cases would suggest.

Calcification of intervertebral discs has been recognized in association with certain metabolic disturbances, particularly alkaptonuria, but in this disease the process is generalized throughout the spine, whereas in the type referred to above it is localized to a maximum of four segments.

The process may affect either the nucleus pulposus or the annulus fibrosus, but the latter is the more common in children. The cervical and thoracic portion of the spine is more frequently affected than the lumbar region.

Symptoms referable to the affected area may or may not be present, but do not appear to bear a close relationship to the development or disappearance of the calcium deposits. Occasionally the lesion has been detected in a routine chest radiograph. Calcification in the cervical region is more likely to be associated with symptoms than when the thoracic or lumbar region is involved.

The symptoms are backache, stiffness of the spine, and occasionally torticollis. Associated congenital abnormalities have been noted in three of the 16 recorded cases but the relationship between the two is not clear. The following case belongs to the group with local symptoms.

\section{Case History}

C.Z., a 5-year-old child, born of Dutch parents, developed some stiffness and pain in the upper part of the back on March 20, 1956. These symptoms were most noticeable in the mornings and tended to improve throughout the day. However, over the next two weeks the stiffness became more marked and the pain more severe and she was admitted to hospital on April 16. There were no other symptoms and she had not been febrile.

Examination on admission showed a healthy looking child, with a normal temperature, crying with pain in her back. She was unable to sit up without assistance and there was marked neck and back stiffness. There was slight tenderness in the upper thoracic spine, but no deformity. All other systems were normal and there were no congenital abnormalities.

The white count was 14,000 of which $60 \%$ were polymorphonuclear leucocytes. The sedimentation rate was 10 millimetres in the first hour and the Mantoux test was negative. A radiograph of the spine showed well marked calcification of the intervertebral discs between

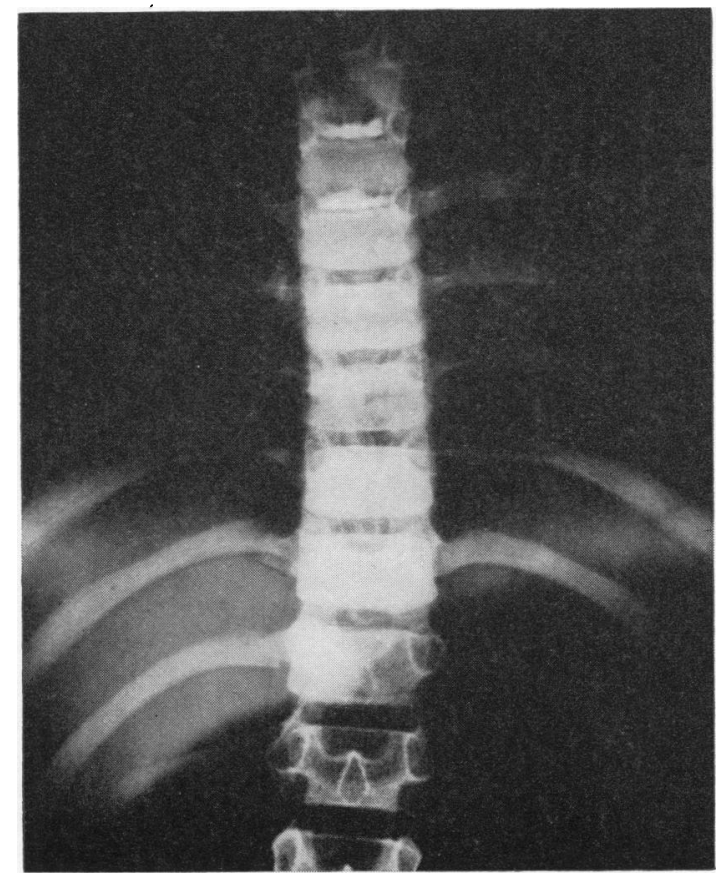

Fig. 1. 


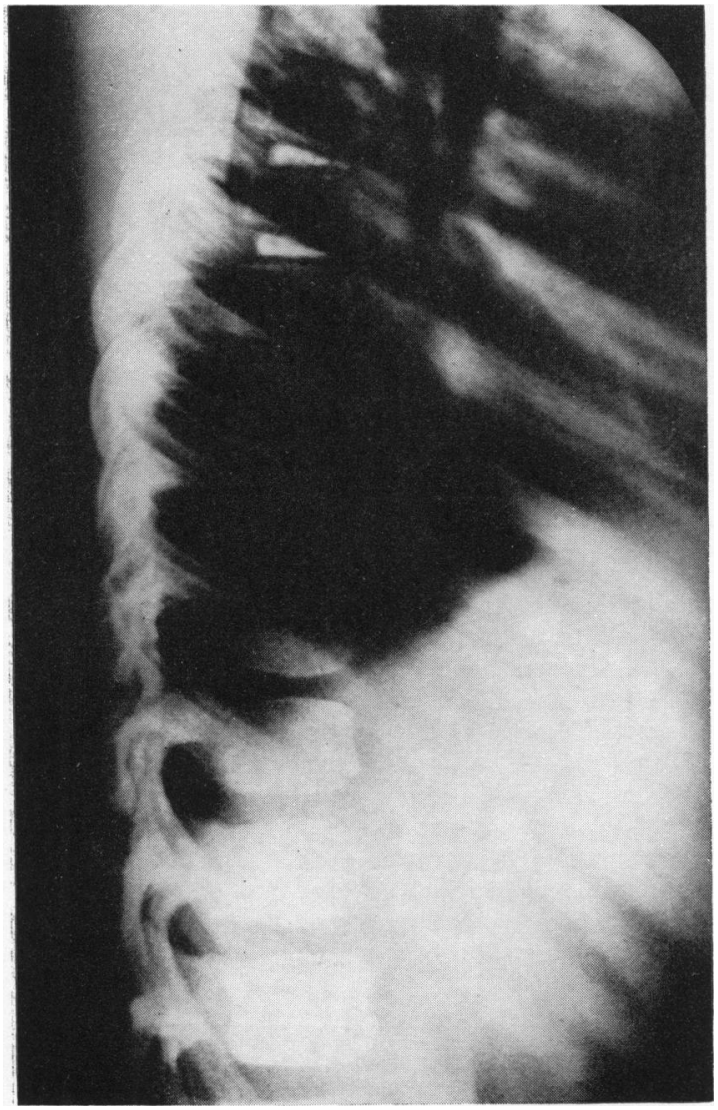

Fig. 2.

the fourth, fifth and sixth thoracic vertebrae (Figs. 1 and 2). No calcification was seen elsewhere. No homogentisic acid was detected in the urine. Calcium and phosphorus estimations were not carried out.

The pain and back stiffness remained marked for three days. Over the succeeding week the symptoms gradually subsided and 10 days after admission she appeared perfectly well and had a completely mobile spine. On August 13 the radiological appearances in the vertebrae were unchanged.

\section{Discussion}

There appears little doubt that there is a relationship between the pain and back stiffness and the radiological changes. However, it is also obvious that the process must have been active long before the onset of the symptoms, because three weeks after the first sign of pain, well marked calcification was seen in the radiograph.

The aetiology of the condition is obscure. The lack of pyrexia and the normal sedimentation rate make an infective basis unlikely, and there was no history of injury to suggest a traumatic aetiology. Degeneration seems untenable in view of the age of the patient. In fact in one of Silverman's (1954) cases calcification was detected as early as 12 months of age.

Whatever the aetiology, the condition appears to be reversible and in reported cases the calcium deposits have been observed to disappear gradually over a period of months to years. No treatment appears indicated apart from rest and analgesics while there are symptoms.

\section{Summary}

A case of calcification of intervertebral discs in a 5-year-old girl is described. Attention is drawn to its infrequency in children, its good prognosis and the reversible nature of the radiological changes.

\section{REFERENCES}

Báron, A. (1924). Jb. Kinderheilk., 104, 357. Quoted by Weens, H. S. (1945). J. Pediat., 26, 178. Silverman, F. N. (1954). Radiology, 62, 801. 